\title{
Der Einfluss einer Schuheinlage aus Karbon auf die plantare Druckverteilung im Radschuh
}

\author{
Heiner Baur, Jan Hoffmann, Anne Reichmuth, Steffen Müller, Frank Mayer
}

\section{Einleitung \\ $\nabla$}

Im Radsport sind Überlastungsbeschwerden ein häufiges Problem. So wird je nach untersuchtem Kollektiv abhängig vom Leistungs- und Aktivitätsniveau der Radsportler über eine Verletzungsinzidenzrate von 21 bis $94 \%$ pro Trainingsjahr berichtet [1-4]. Neben Rückenbeschwerden ist insbesondere die Knieregion (Iliotibiales Bandsyndrom, vorderer Knieschmerz) betroffen, die bei professionellen Radsportlern am häufigsten mit Trainingsreduktion und Trainingsausfall assoziiert ist [3]. Da die einwirkenden Kräfte auf die untere Extremität im Vergleich zum Gehen und Laufen geringer eingestuft werden, wird vermutet dass möglichweise die repetitive Charakteristik der Belastung problematischer für die Beschwerdeentstehung einzuschätzen ist, als die Höhe der Belastung selbst [5]. Fußbezogene Beschwerden im Radsport sind Metatarsalgien, Achillessehnenbeschwerden und häufig durch Schuhe und deren Verschlussmechanismen hervorgerufene Taubheitsgefühle im Fußbereich [6, 7]. Die Entstehung von Überlastungsbeschwerden wird einer nicht optimalen Position auf dem Rad zugeschrieben [8, 9]. In Reviews werden zwar Maßnahmen zur Reduktion von überlastungsbedingten Beschwerden vorgeschlagen (Sitzposition optimieren [9], Schuh-Pedal-Interface optimieren [8], Fußposition ändern, um Bewegungsablauf zu optimieren [10-12]). Allerdings gibt es keine Studien, welche die Effektivität dieser Optimierungsstrategien zur Reduktion von Beschwerden oder deren Präventionspotenzial im Längsschnitt überprüfen [13]. Lediglich wenige Querschnittsuntersuchungen zu biomechanischen Effekten durch Radschuheinlagen sind bisher publiziert.

Eine aktuelle Studie analysierte Einflüsse verschiedener Fußbettungen (Varuskeile in Vor- oder Rückfuß) auf die Kinematik der unteren Extremität während der Pedalierbewegung auf dem Rad. Die Ergeb- nisse zeigten im interindividuellen Vergleich keine systematischen Effekte. Lediglich ein statistisch nicht signifikanter „Trend“ hinsichtlich einer reduzierten Tibiainnenrotation durch Schuheinlagen konnte konstatiert werden. Intraindividuelle subjektspezifische Unterschiede zwischen den Einlagenkonditionen wurden jedoch verzeichnet und es wird deshalb ein individuelles Vorgehen in der Hilfsmittelversorgung von Radsportlern vorgeschlagen [12]. Eine erhöhte lokale Spitzendruckbelastung im Fuß wurde mit der Entstehung von Metatarsalgien und anderen fußbezogenen Beschwerden in Verbindung gebracht. Die Steifheit der Schuhsohle sowie die Konfiguration der Schuh/ Pedal-Verbindung seien dabei beeinflussende Faktoren für die Druckbelastung des Fußes [8]. Jarboe \& Quesada (2003) untersuchten deshalb die Effekte unterschiedlicher Schuhkonstruktionen (Karbonsohle vs. Kunststoffsohle) und konnten im Schuh mit der objektiv steiferen Sohle aus Karbon höhere Spitzendruckbelastungen im Vorfuß (+18\%) im Vergleich zum Schuh mit Kunststoffsohle messen [14]. Gleichzeitig wird ein steifes Interface zwischen Fuß und Pedal (Schuh/Einlagenkombination, Orthese, Prothese, etc.) benötigt, um die entwickelte Kraft des Körpers möglichst ideal auf das Pedal übertragen zu können $[15,16]$.

Eine individualisierte Schuheinlagenversorgung sollte deshalb im Idealfall zusammen mit dem Schuh ein steifes Interface zwischen Fuß und Pedal bilden, gleichzeitig jedoch nicht zu einer erhöhten Druckbelastung direkt am Fuß führen. Dies sind spezifische Anforderungen an Material und Formgebung einer Radschuheinlage. Der Werkstoff Karbon wird in Schuhen für das normale Gehen zur Versteifung des Vorfußes eingesetzt [17]. Das sehr steife und dennoch leichte Material führt zu einer Gewichtsreduktion der Hilfsmittelversorgung [18]. Eine daraus gefertigtes Hilfsmittel ist dauerhaft einsetzbar und sehr widerstandsfähig, führt dabei jedoch zu Mehrkosten in der Herstellung [19, 20]. Bisher beschäftigten sich Studien hauptsächlich mit dem Einsatz von Karbon bei Orthesenversorgungen [19, 21]. Noch nicht geklärt ist jedoch, ob Karbon als Werkstoff auch bei Schuheinlagen im Radschuh eingesetzt werden kann. Ziel der Untersuchung war deshalb die Analyse der Spitzendruckbelastung unter dem Fuß bei der Anwendung einer Radschuheinlage aus Karbon im Vergleich zu einer herkömmlichen Standardeinlegesohle.

\section{Material und Methoden $\nabla$ \\ Probanden}

In die Studie wurden 11 gesunde männliche Probanden eingeschlossen (29 \pm 9 Jahre, $1,77 \pm 0,04 \mathrm{~m}, 68 \pm 5 \mathrm{~kg}$ ). Alle Probanden betreiben Triathlon als Wettkampfsportart und verfügen über einen wöchentlichen Radtrainingsumfang von 6,2 $\pm 3,6 \mathrm{~h}$. Dazu kommen 9,0 $\pm 6,2$ h Alternativtraining (Langstreckenlauf, Schwimmen, allgemeines Athletiktraining). Die mittlere Fußlänge betrug links $0,262 \pm 0,008 \mathrm{~m}$ und rechts $0,262 \pm 0,009 \mathrm{~m}$. Dies entspricht Schuhgröße EU 43. Der Einschluss erfolgte freiwillig. Es wurde von allen Teilnehmern eine „Informed Consent Form“ unterschrieben, die nach GCP-Richtlinien und Vorgaben der universitären Ethikkommission erstellt wurde. Ein internistischer und orthopädischer Check-up schloss Beschwerden und akute Infekte aus. Somit waren alle Probanden fähig das Testprotokoll zu absolvieren.

\section{Protokoll}

Die Probanden wurden auf einem Fahrradergometer (Lode Excalibur Sport, Lode B.V., Groningen, NL) mit einem ihrer Fußlänge entsprechenden Standardradschuh in Größe 43 (Sidi Genius 4, Sidi Sport Srl, Maser/Treviso, IT) mit Klickpedalfixierung getestet (System SPD, Pedal PDM324 mit Doppelfunktion, Shimano, Sakai, Osa- 


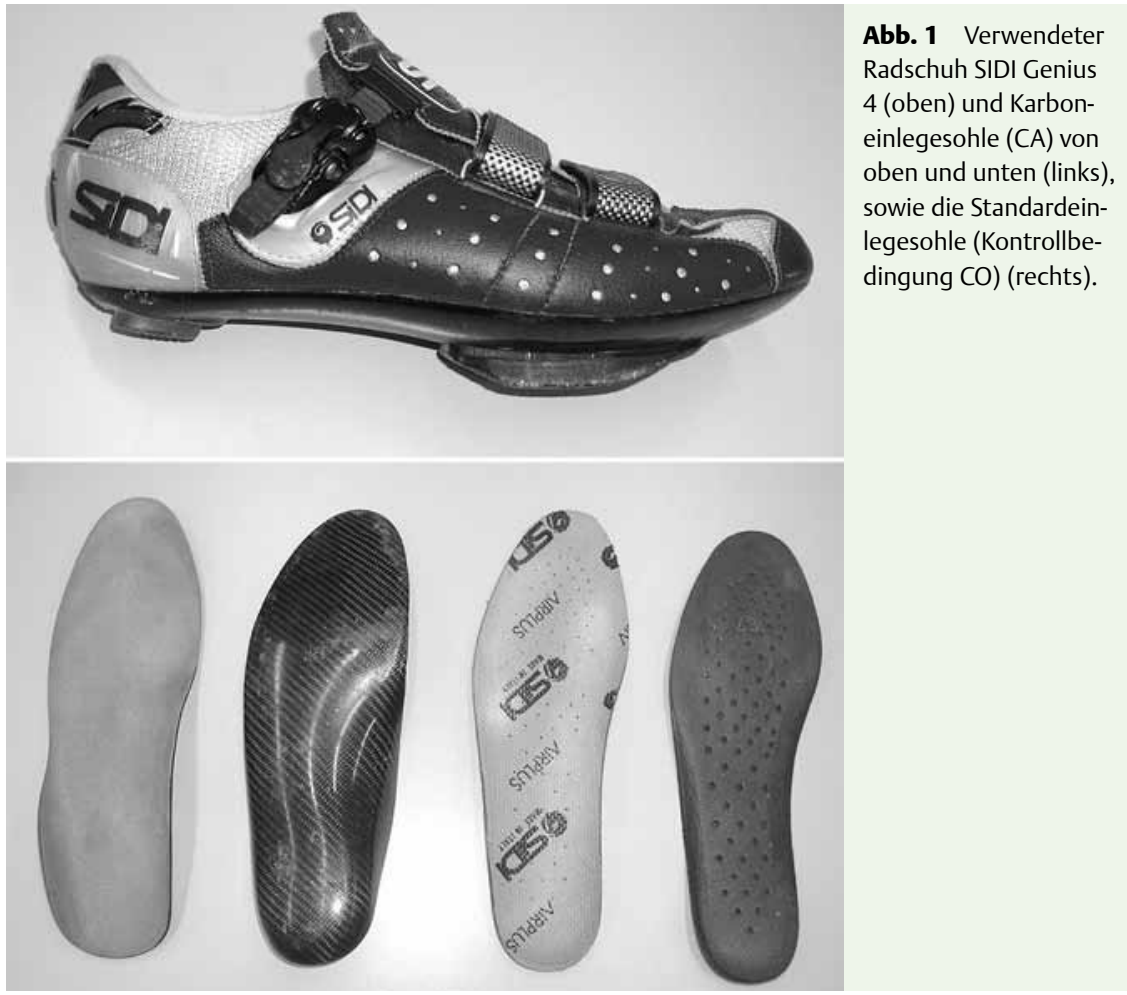

ka, JP). In randomisierter Reihenfolge trugen die Probanden entweder den Schuh mit der Standardeinlegesohle aus perforiertem thermoplastisch geformtem EVA ( $\triangle$ Abb.1 Kontrollkondition CO) oder den Schuh mit einer Schuheinlegesohle aus Karbon (Kondition CA) (Technische Orthopädie Volkery, Unna, DE). Die Karbonsohle wurde im Prepreg-Verfahren aus 6 Lagen Karbonfaser (Easypreg, Exact Plastics, Bröckel, DE) mit 4 mulitdirektionalen Glasfaserzwischenlagen über einen Standardleisten geformt. Die Karbonlagen sind primär im Verlauf der Kraftübertragung angeordnet. Sie nehmen gestaffelt lagenweise in Richtung Vorfuß ab und richten sich zum lateralen Fußrand aus. Eine Schalenform in der Ferse, eine Längsgewölbeanhebung (30 mm) und eine leichte Außenranderhöhung im Vorfuß (3mm) bildeten Funktionselemente in der Karbonsohle. Ein Alcantara-Microfaserbezug ( $2 \mathrm{~mm}$ ) bildet die Kontaktfläche zum Fuß. Diese Experimentalkondition ist bezüglich der Sprengung an den Schuh angepasst und verfügt über die gleiche Materialstärke wie die Standardeinlegesohle (4 mm) und führt damit nicht zu einer Veränderung des Schuhinnenvolumens.

Der Fahrradergometer wurde zunächst bezüglich der Sitzposition von jedem Probanden individuell analog der Sitzposition auf dem Trainingsrad adjustiert. Danach erfolgte eine standardisierte Erwärmung (Leistung/Intensität: 80 Watt [W],
Trittfrequenz 90 „rotations per minute“ [rpm], Dauer 15 Min.). Anschließend absolvierten die Probanden je Schuhkondition 2 Tests bei $200 \mathrm{~W}$ (bei einer Trittfrequenz von 60 und 90 rpm und 2 Tests bei $300 \mathrm{~W}$ [ebenfalls bei 60 und $90 \mathrm{rpm}$ ] in jeweils randomisierter Abfolge. Abschließend erfolgte ein 3-minütiges Cool-down bei $50 \mathrm{~W}$ und $90 \mathrm{rpm}$. Während der Tests wurde bei konstanter Trittfrequenz jeweils für 25 s die plantare Druckverteilung im Schuh gemessen (pedar-X, Novel, München, DE). Das normalerweise zur Gang- und Laufanalyse verwendete InSchuh-Sohlenmesssystem wurde aufgrund der zu erwartenden niedrigeren Spitzendruckbelastungen speziell kalibriert. Analog zu dem von Jarboe et al. 2003 vorgeschlagenen Procedere wurden mithilfe der Kalibrationseinheit des Herstellers (trublu, Novel, München, DE) die Messsohlen für einen Messbereich von 0-250 kPa kalibriert (vgl.: vorgeschlagener Kalibrationsbereich für die Ganganalyse: 0-600 kPa) [14, 15, 22].

Nach der Datenaufzeichnung mit der Herstellersoftware (pedar-X-expert, Version 11.3.12, Novel, München, DE) erfolgte ein ASCII-Export der Daten. Ein speziell konfiguriertes Datenblattmodul (Microsoft Excel, Redmond, US) erlaubte das Auslesen der Spitzendruckwerte pro Trittzyklus. Als Messgröße diente der gemittelte Spitzendruck aus 30 Trittzyklen. Dieser Mittelwert wurde pro Proband und Mess- kondition für den Gesamtfuß, den Rückfuß, den Mittelfuß, den Vorfuß (gesamter Vorfuß; lateraler Vorfuß, zentraler Vorfuß, medialer Vorfuß) und den Zehenbereich erfasst und in eine Statistikdatenbank überführt (JMP Version 5.0.1, Cary, US).

Die Ergebnisse werden deskriptiv (Mittelwert, Standardabweichung, Upper und Lower 95\%-Konfidenzintervall) dargestellt. Mittels dreifaktoriellen ANOVAs (Kondition, Trittfrequenz, Leistung) mit Messwertwiederholung $(\alpha=0,05)$ wurde der Einfluss der Einlagenbedingung auf die Spitzendruckbelastung im Gesamtfuß (Hauptfragestellung) und in verschiedenen Fußarealen (Rückfuß, Mittelfuß, Vorfuß, Zehenbereich) geprüft.

\section{Ergebnisse}

Zunächst wurde untersucht, ob möglicherweise Seitenunterschiede zwischen linker und rechter Extremität auftraten. Dies war nicht der Fall $(p=0,36)$. Deshalb beinhaltet die folgende Ergebnisdarstellung die gemeinsame Betrachtung von Spitzendruckwerten beider Extremitäten.

\section{Gesamtfuß}

Für den Gesamtfuß ergaben sich im Mittel bei $200 \mathrm{~W}$ Spitzendruckwerte im Bereich von $70-75 \mathrm{kPa}$. Bei $300 \mathrm{~W}$ lagen die Spitzendruckwerte im Bereich von 85$110 \mathrm{kPa}(\odot \mathbf{A b b}$. 2). Die Spitzendruckbelastung war mit der Karbonsohle um 4,1\% gegenüber der Standardeinlegesohle geringer. Die Analyse der Hauptfragestellung nach dem Einfluss der Karboneinlage auf den Spitzendruck im Gesamtfuß zeigte im Vergleich zur Standardeinlegesohle (Faktor Einlage) jedoch keinen statistisch signifikanten Unterschied $(p=0,10$, -Tab.1). Innerhalb der dreifaktoriellen ANOVA ergaben sich signifikante Effekte für die beiden Faktoren Leistung und Trittfrequenz: Die Spitzendruckwerte bei 300 W lagen höher als bei 200W $(p<0,001)$ und bei $60 \mathrm{rpm}$ waren die Spitzendruckwerte ebenfalls höher als bei $90 \mathrm{rpm}$ $(\mathrm{p}<0,001)(\diamond \mathbf{A b b . 2})$.

\section{Rück-, Mittel-, Vorfuß und Zehenbereich}

Bei deskriptiver Betrachtung der verschiedenen Fußareale wurden im Vorfuß mit mittleren Spitzendruckbelastungen im Bereich von 79 bis $85 \mathrm{kPa}$ die höchsten 

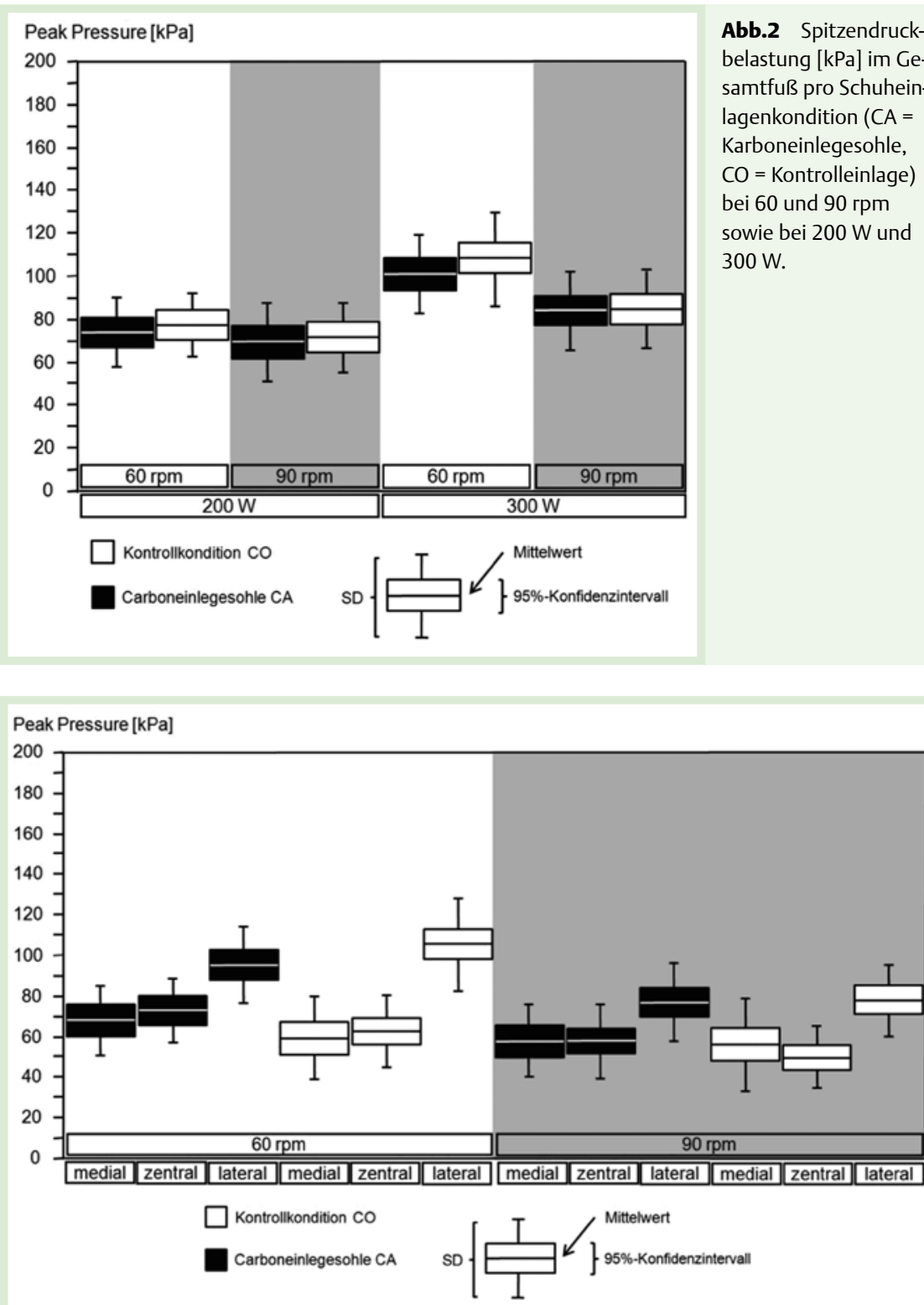

Abb. 3 Spitzendruckbelastung [ $\mathrm{kPa}]$ in den Vorfußarealen medialer Vorfuß, zentraler Vorfuß, lateraler Vorfuß pro Schuheinlagenkondition ( $C A=$ Karboneinlegesohle, $\mathrm{CO}=$ Kontrolleinlage $)$ bei 60 und $90 \mathrm{rpm}$ und $300 \mathrm{~W}$.
Abb.2 Spitzendruckbelastung $[\mathrm{kPa}] \mathrm{im} \mathrm{Ge}-$ samtfuß pro Schuheinlagenkondition ( $\mathrm{CA}=$ Karboneinlegesohle, $\mathrm{CO}=$ Kontrolleinlage) bei 60 und $90 \mathrm{rpm}$ sowie bei $200 \mathrm{~W}$ und $300 \mathrm{~W}$.

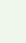

Spitzendruckwerte verzeichnet. Daneben ist der Zehenbereich als die am zweithöchsten belastete Region, mit Werten von circa 51 bis $59 \mathrm{kPa}$ bereits erheblich weniger Druckbelastung ausgesetzt. Dagegen ist der Mittelfußbereich mit 38$47 \mathrm{kPa}$ und der Rückfuß mit Werten von 26 bis $31 \mathrm{kPa}$ nur gering belastet ( 0 Tab.1).

Die differenzierte Analyse in den verschiedenen Fußbereichen zeigte im Gegensatz zur Analyse im Gesamtfuß jeweils statistisch signifikante Unterschiede für den Faktor Einlage ( $\bullet$ Tab. 1). Im Rückfuß ergab sich eine Spitzendruckreduktion von $16,6 \%$ durch die Karboneinlage, ( $p<0,001)$. Im Mittelfuß sorgte die Experimentalkondition CA für eine Reduktion der Spitzendruckbelastung um $20 \%$. Der Spitzendruck in dem am höchsten belasteten Vorfußbereich war bei Einsatz der Karboneinlage um 5,9\% reduziert ( $p=0,03$ ). Dagegen erhöhte sich die Spitzendruckbelastung im Zehenbereich durch die Karboneinlage um $16,2 \%(p<0,001)$ ( $\odot$ Tab. 1).

\section{Vorfuß differenziert}

Bei differenzierter Betrachtung des Vorfuß zeigt sich unabhängig von der Einlagenkondition, der Trittfrequenz oder der Leistung eine nahezu gleich hohe Spitzendruckbelastung für den medialen und den zentralen Vorfuß. Demgegenüber ist der laterale Vorfuß erhöhter Druckbelastung ausgesetzt. Die $\odot \mathbf{A b b}$. 3 verdeutlicht dies in der Darstellung der Spitzendruckwerte pro Kondition und Trittfrequenz bei der getesteten Leistung von $300 \mathrm{~W}$.

Der prozentuale Anstieg der Spitzendruckwerte von medial und zentral nach lateral beträgt bei der Karboneinlegesohle im Mittel 34\% und bei der Kontrollkondition im Mittel 59\%.

\section{Diskussion}

$\nabla$

Die Untersuchung prüfte den Einfluss einer Schuheinlage aus Karbon auf die Spitzendruckbelastung des Fußes im Radschuh während des Pedalierens. Dies wurde im Vergleich zu einer herkömmlichen Standardeinlegesohle eines handelsüblichen Radschuhs getestet. Die Spitzendruckbelastung im Gesamtfuß war mit Karboneinlegesohle um 4\% gegenüber der Standardeinlegesohle reduziert, ohne jedoch statistische Signifikanz aufzuweisen. 
Hintergrund: Zur Reduktion von Überlastungsbeschwerden im Radsport werden verschiedene Equipment-Interventionen (Optimierung Sitzposition, Optimierung Schuh/Einlage/Pedalkombination, etc.) empfohlen. Allerdings fehlen weitgehend Studien über klinische oder biomechanische Effekte der genannten Maßnahmen. Schuheinlagen aus Karbon können eine Möglichkeit sein, das Interface zwischen Fuß und Pedal zu optimieren. Ziel der Studie war deshalb die Analyse der plantaren Druckverteilung unter dem Fuß bei der Anwendung einer Radschuheinlage aus Karbon im Vergleich zu einer herkömmlichen Standardeinlegesohle. Material und Methoden: 11 beschwerdefreie Triathleten (29 \pm 9 Jahre, $1,77 \pm 0,04 \mathrm{~m}, 68 \pm 5 \mathrm{~kg}$ ) wurden auf einem Radergometer bei einer Trittfrequenz von 60 und $90 \mathrm{rpm}$ („rotations per minute“) bei $200 \mathrm{~W}$ und 300W gemessen. Dabei trugen die Probanden in randomisierter Reihenfolge einen Radschuh mit handelsüblicher Einlegesohle (Kontrolle CO) oder den Radschuh mit einer Schuheinlage aus Karbon (CA). Erfasst wurde der mittlere Spitzendruck aus 30 Trittzyklen im Gesamtfuß und in einzelnen Fußregionen (Rück-, Mittel-, Vorfuß [medial, zentral, lateral] und Zehenbereich). Dreifaktorielle ANOVAs (Faktor Einlage, Trittfrequenz, Leistung) mit Messwertwiederholung $(\alpha=0,05)$ dienten zur Prüfung der Hauptfrage nach dem Einfluss der Einlagenkondition auf die plantare Spitzendruckbelastung. Ergebnisse: Die Spitzendruckbelastung im Gesamtfuß lag im Mittel bei 200 Watt (W) im Bereich von 70-75 kPa (300 W: 85-110 kPa). Die Spitzendruckbelastung war mit der Karbonsohle um 4,1\% gegenüber der Standardeinlegesohle reduziert. $(p=0,10)$. Bei Betrachtung der spezifischen Fußareale war der Spitzen- druck bei CA im Rück- $(-16,6 \%, \mathrm{p}<0,001)$, Mittel- $(-20,0 \%, \mathrm{p}<0,001)$ und Vorfuß $(-5,9 \%, p<0,03)$ gegenüber CO verringert. Im Zehenbereich war der Spitzendruck in CA gegenüber $\mathrm{CO}$ um $+16,2 \%$ erhöht $(\mathrm{p}<0,001)$. Innerhalb des Vorfuß zeigte sich der Spitzendruck im lateralen Vorfuß sowohl bei CA (+34\%) als auch bei $\mathrm{CO}(+59 \%)$ gegenüber dem medialen und zentralen Vorfuß erhöht. Schlussfolgerung: Karbon kann als Fertigungswerkstoff in der Schuheinlagenversorgung im Radsport eingesetzt werden. Die plantare Fußsohle wird durch das steife Material gegenüber einer Standardeinlegesohle nicht übermäßig belastet. Bei individueller Anpassung an die Fußform können möglicherweise Fußregionen bei Bedarf sogar entlastet werden. Schlüsselwörter: In-Schuh Sohlenmesssystem, Karbon, Plantare Druckverteilung, Radsport, Schuheinlage

Influence of Carbon Fiber Foot Orthoses on Plantar Pressure Distribution in Cycling

Background: Several equipment interventions like optimizing seat position or optimizing shoe/insole/pedal interface are suggested to reduce overuse injury in cycling. Data analyzing clinical or biomechanical effects of those interventions is sparse. Foot orthoses out of carbon fiber are one possibility to alter the interface between foot and pedal. The aim of this study was therefore to analyze plantar pressure distribution in carbon fiber foot orthoses in comparison to standard insoles of commercially available cycling shoes. Materials and Methods: 11 painfree triathletes (Age: $29 \pm 9,1.77 \pm 0.04 \mathrm{~m}$, $68 \pm 5 \mathrm{~kg}$ ) were tested on a cycle ergometer at 60 and 90 rotations per minute (rpm) at workloads of 200 and 300 Watts. Sub- jects wore in randomized order a cycling shoe with its standard insole (control condition $\mathrm{CO}$ ) or the shoe with carbon fiber foot orthoses (Condition CA). Mean peak pressure out of 30 movement cycles were extracted for the total foot and specific foot regions (rear, mid, fore foot (medial, central, lateral) and toe region). Three-factor ANOVAs (factor foot orthoses, rpm, workload) for repeated measures $(\alpha=0.05)$ were used to analyze the main question of a foot orthoses effect on peak in-shoe plantar pressure. Results: Peak pressures in the total foot were in a range of $70-75 \mathrm{kPa}$ for 200 Watts (W) (300 W: $85-110 \mathrm{kPa}$ ). The carbon fiber foot orthoses reduced peak pressures by $-4,1 \%$ compared to the standard insole $(\mathrm{p}=0,10)$. In the foot regions rear $(-16,6 \%$, $\mathrm{p}<0.001)$, mid $(-20,0 \%, \mathrm{p}<0.001)$ and fore foot $(-5.9 \%, \mathrm{p}<0.03)$ CA reduced peak pressure compared to $\mathrm{CO}$. In the toe region, peak pressure was higher in $\mathrm{CA}$ $(+16,2 \%)$ compared to $\mathrm{CO}(\mathrm{p}<0,001)$. The lateral fore foot showed higher peak pressures in CA (+34\%) and CO (+59\%) compared to medial and central fore foot. Conclusion: Carbon fiber can serve as a suitable material for foot orthoses manufacturing in cycling. Plantar pressures do not increase due to the stiffness of the carbon. Individual customization may have the potential to reduce peak pressure in certain foot areas. Keywords: Carbon, Cycling, Foot orthoses, In-shoe measurement, Plantar Pressure Distribution
Die gemessenen Spitzendruckwerte liegen bei $300 \mathrm{~W}$ mit 95-110 kPa etwas unter den in der Literatur beschriebenen Vergleichswerten von Jarboe \& Quesada (2003) [14]. Dort wurde eine Range von 103 bis $121 \mathrm{kPa}$ gemessen. Allerdings wurde dort auch bei einer Leistung von $400 \mathrm{~W}$ geprüft. In dieser Untersuchung wurden auch keine Seitendifferenzen festgestellt [14]. Die Reduktion der Spitzendruckwerte bei gleichbleibender Leistung und höherer Trittfrequenz erscheint plausibel, da bei gleichbleibender belaste- ter Fläche unter dem Fuß die notwendige Kraft pro Trittzyklus sinkt. Ebenso ist der Anstieg der Spitzendruckbelastung bei gleichbleibender Trittfrequenz und Erhöhung der Leistung (von $200 \mathrm{~W}$ nach 300 W) folgerichtig, da pro Trittzyklus mehr Kraft auf das Pedal gebracht werden muss. Die vorliegenden Messungen spiegeln damit grundlegende physikalische Gesetzmäßigkeiten wider und bestätigen Befunde in der Literatur [23]. Es zeigte sich ebenfalls, dass im Vorfuß relevante Spitzendrücke entstehen und die anderen
Fußregionen weit weniger belastet werden $[14,15,23]$. Die Daten der vorliegenden Untersuchung können somit als valide bewertet werden.

Bei Betrachtung der einzelnen Fußregionen zeigt sich im Rück-, Mittel-, und Vorfuß jeweils eine Reduktion der Belastung durch die Karboneinlegesohle im Vergleich zur normalen Standardeinlegesohle. Dies kann möglicherweise, zumindest in Rück- und Mittelfuß, an der dreidimensionalen Ausformung der Interventions- 
einlage liegen (Schalenform im Rückfuß, Längsgewölbeanhebung im Mittelfuß). Die Belastung wird dadurch auf eine größere Fläche verteilt, was die Spitzendruckbelastung sinken lässt. Eine Anformung der Schuheinlage an die individuelle Fußform kann diesen Effekt möglicherweise verstärken. Obwohl die Effekte durch die Karboneinlage zu einem statistisch signifikanten Unterschied führen, kann angemerkt werden, dass die Unterschiede im Bereich von $<20 \%$ sind und somit deren klinische Relevanz kritisch zu hinterfragen ist.

Die Karboneinlage sorgte bei differenzierter Betrachtung der Vorfußregionen für einen leichten Ausgleich der Druckverteilung zwischen medialem, zentralem und lateralem Vorfuß, wenngleich die gleiche Charakteristik mit höchster Belastung im lateralen Vorfuß wie mit der Standardsohle erhalten bleibt. Dies steht im Gegensatz zu den Ergebnissen von Sanderson et al. (2000). Dort wurden die höchsten Belastungen unter MFK I gemessen [23]. Im Sinne einer effektiven Übertragung der Kraft auf das Pedal ist mit Sicherheit die höchste Belastung im medialen Vorfuß anzustreben. Dabei muss der intrinsische Zusammenhang von Fuß(form), Schuh(form) und Pedalkonfiguration beachtet werden [23]. Eine individualisierte Versorgung mit Abformung der Vorfußform unter Last kann das angestrebte Belastungsprofil erreichen [15].

Vergleicht man die gemessenen Spitzendruckwerte im Radschuh mit anderen Belastungsformen, so sind in absoluter Hinsicht die Spitzendruckwerte in beiden Schuhkonditionen als gering einzustufen. Die Werte sind vergleichbar mit Spitzendrücken wie sie bei Kleinkindern im Gang auftreten [24]. Bei Erwachsenen können im Gehen und Laufen bis zum mehr als 3-Fachen der Spitzendruckwerte im Schuh auftreten $[25,26]$. Bei dynamischen Bewegungen (run, cut, sprint, kick) steigen die Druckwerte sogar bis zum 6-Fachen der Druckbelastung im Radschuh [27]. Es kann daher die Frage gestellt werden, ob die im Radschuh bei mittlerer Leistung gemessenen Werte trotz des repetitiven Charakters ursächlich für Beschwerden sind [5]. Dies ist im besten Fall durch prospektive Daten zu beantworten, die bisher nicht verfügbar sind.

Zu den Limitationen der Studie gehört sicher der moderate Workload-Bereich in dem gemessen wurde (200-300 W). Zu- mindest kurzfristig können sowohl im Mountainbikesport, im Straßenradsport und insbesondere im Bahnradsport weit höhere abgegebene Leistungen resultieren [28-30]. Gleiches gilt für die gemessenen Trittfrequenzen, die insbesondere in Sprintsituationen und auf der Radbahn weit über die gemessenen 90 rpm hinausgehen [29]. Die Aussagekraft der Ergebnisse für den Freizeitradsport bleibt dennoch erhalten. Eine weitere Limitation der Untersuchung ist die Intervention mit einer standardisierten Karboneinlagenkondition. Differenzierte Ergebnisse sind sicher durch die Untersuchung individualisierter Hilfsmittelversorgungen zu erwarten. Die Studie soll jedoch ein erster Schritt zur Abschätzung des Potenzials von Karbon als Werkstoff für Radschuheinlagen sein.

\section{Schlussfolgerung \\ $\nabla$}

Karbon kann als Werkstoff für Radschuheinlagenversorgungen gut eingesetzt werden. Die plantare Fußsohle wird durch das steife Material nicht übermäßig belastet. Bei individueller Anpassung an die Fußform können möglicherweise Fußregionen bei Bedarf sogar entlastet werden.

\section{Interessenkonflikt}

$\nabla$

Es besteht kein Interessenkonflikt

Literatur

1 Dannenberg AL, Needle S, Mullady Det al. Predictors of injury among 1638 riders in a recreational long-distance bicycle tour: Cycle Across Maryland. Am J Sports Med 1996; 24 : 747-753

2 Wilber CA, Holland GJ, Madison RE et al. An epidemiological analysis of overuse injuries among recreational cyclists. Int J Sports Med 1995; 16: 201-206

3 Clarsen B, Krosshaug T, Bahr R. Overuse injuries in professional road cyclists. Am J Sports Med 2010; 38: 2494-2501

4 Weiss BD. Nontraumatic injuries in amateur long distance bicyclists. Am J Sports Med 1985; 13: 187-192

5 Farrell KC, Reisinger KD, Tillman MD. Force and repetition in cycling: possible implications for iliotibial band friction syndrome. Knee 2003; 10: 103-109

6 Schwellnus MP, Derman EW. Common injuries in cycling: prevention, diagnosis and management. SA Fam Pract 2005; 47: 14-19

7 Mellion MB. Common cycling injuries. Management and prevention. Sports Med 1991; 11: 52-70

8 Gregor RJ, Wheeler JB. Biomechanical factors associated with shoe/pedal interfaces. Im- plications for injury. Sports Med 1994; 17: 117-131

9 Silberman MR, Webner D, Collina S et al. Road bicycle fit. Clin J Sport Med 2005; 15: 271276

10 Faria IE. Applied physiology of cycling. Sports Med 1984; 1: 187-204

11 Sanner WH, O'Halloran WD. The biomechanics, etiology, and treatment of cycling injuries. J Am Podiatr Med Assoc 2000; 90: 354376

12 O`Neill BC, Graham K, Moresi M et al. Custom formed orthoses in cycling. J Sci Med Sport 2011; 14: 529-534

13 Dettori NJ, Norvell DC. Non-traumatic bicycle injuries : a review of the literature. Sports Med 2006; 36: 7-18

14 Jarboe NE, Quesada PM. The effects of cycling shoe stiffness on forefoot pressure. Foot Ankle Int 2003; 24: 784-788

15 Baur H, Stapelfeldt B, Hirschmuller A et al. Functional benefits by sport specific orthoses in a female paralympic cyclist: a case report. Foot Ankle Int 2008; 29: 746-751

16 Childers WL, Kistenberg RS, Gregor RJ. Pedaling Asymmetries in Cyclists with Unilateral Transtibial Amputation: Effect of Prosthetic Foot Stiffness. J Appl Biomech 2011; 27: 314-321

17 Ibuki A, Bach T, Rogers D et al. An investigation of the neurophysiologic effect of tone-reducing AFOs on reflex excitability in subjects with spasticity following stroke while standing. Prosthet Orthot Int 2010; 34: 154-165

18 Granata C, De Lollis A, Campo Get al. Analysis, design and development of a carbon fibre reinforced plastic knee-ankle-foot orthosis prototype for myopathic patients. Proc Inst Mech Eng H 1990; 204: 91-96

19 Brehm MA, Beelen A, Doorenbosch CA et al. Effect of carbon-composite knee-ankle-foot orthoses on walking efficiency and gait in former polio patients. J Rehabil Med 2007; 39: 651-657

20 Hachisuka K, Arai K, Arai M. Carbon fibre reinforced plastic knee-ankle-foot orthosis with a partially flexible thigh cuff: a modification for comfort while sitting on a toilet seat. Prosthet Orthot Int 2007; 31: 133-137

21 Faustini MC, Neptune RR, Crawford RH et al. Manufacture of Passive Dynamic ankle-foot orthoses using selective laser sintering. IEEE Trans Biomed Eng 2008; 55: 784-790

22 Rozema A, Ulbrecht JS, Pammer SE et al. Inshoe plantar pressures during activities of daily living: implications for therapeutic footwear design. Foot Ankle Int 1996; 17 : 352-359

23 Sanderson DJ, Hennig EM, Black AH. The influence of cadence and power output on force application and in-shoe pressure distribution during cycling by competitive and recreational cyclists. J Sports Sci 2000; 18: 173-181

24 Bosch K, Gerss J, Rosenbaum D. Development of healthy children's feet-nine-year results of a longitudinal investigation of plantar loading patterns. Gait Posture 2010; 32: 564571 
25 Fiedler KE, Stuijfzand WJ, Harlaar J et al. The effect of shoe lacing on plantar pressure distribution and in-shoe displacement of the foot in healthy participants. Gait Posture 2011; 33: 396-400

26 Rethnam U, Makwana N. Are old running shoes detrimental to your feet? A pedobarographic study. BMC Res Notes 2011; 4: 307

27 Eils E, Streyl M, Linnenbecker S et al. Characteristic plantar pressure distribution patterns during soccer-specific movements. Am J Sports Med 2004; 32: 140-145
28 Stapelfeldt B, Schwirtz A, Schumacher YO et al. Workload demands in mountain bike racing. Int J Sports Med 2004; 25: 294-300

29 Craig NP, Norton KI. Characteristics of track cycling. Sports Med 2001; 31: 457-468

30 Vogt S, Roecker K, Schumacher YO et al. Cadence-power-relationship during decisive mountain ascents at the Tour de France. Int J Sports Med 2008; 29: 244-250

\section{Heiner Baur ${ }^{1,2}$ \\ Jan Hoffmann ${ }^{1}$ \\ Anne Reichmuth ${ }^{1}$ \\ Steffen Müller ${ }^{1}$ \\ Frank Mayer ${ }^{1}$}

\section{${ }^{1}$ Hochschulambulanz der Universität} Potsdam, Sportmedizin und Sportorthopädie, Universität Potsdam, Deutschland ${ }^{2}$ Berner Fachhochschule, Gesundheit, aFGE Physiotherapie, Bern, Schweiz

Korrespondenzadresse:

Heiner Baur

Bern University of Applied Sciences Health, aR\&D Physiotherapy Murtenstrasse 10

CH-3008 Bern, Switzerland E-Mail: heiner.baur@bfh.ch 